Conclusions Disease activity, fibromyalgia and ongoing therapy all contribute to determine SLE burden. In particular, arthritis and skin disease have a great impact on patient daily living. Moreover, steroid therapy negatively influences patient perception of the disease.

Funding Source(s): Founding: INTEGRATE project (European Commission, 3rd Health Program, Proposal ID 769736)

\section{BARICITINIB-ASSOCIATED CHANGES IN TYPE I INTERFERON GENE SIGNATURE DURING A 24-WEEK PHASE 2 CLINICAL SLE TRIAL}

${ }^{1}$ Thomas Dörner, ${ }^{2}$ Yoshiya Tanaka, ${ }^{3}$ Michelle Petri, ${ }^{4}$ Josef S Smolen, ${ }^{5}$ Ernst R Dow, ${ }^{5}$ Richard E Higgs, ${ }^{5}$ Robert J Benschop, ${ }^{5}$ Adam Abel, ${ }^{5}$ Maria E Silk, ${ }^{5}$ Stephanie de Bono, ${ }^{5}$ Robert W Hoffman*. 'Charite Universitätsmedizin Berlin and Deutsches RheumaForschungszentrum (DRFZ); ${ }^{2}$ University of Occupational and Environmental Health, Japan; ${ }^{3}$ Johns Hopkins University School of Medicine; ${ }^{4}$ Medical University of Vienna; ${ }^{5}$ Eli Lilly and Company

\subsection{6/lupus-2019-Ism.185}

Background In the phase 2 study JAHH (NCT02708095), treatment with baricitinib, an oral selective Janus kinase $1 / 2$ inhibitor approved for the treatment of rheumatoid arthritis, resulted in significant improvements in patients with active SLE receiving standard background therapy compared with placebo. ${ }^{1}$ Expression of type I-associated interferon (IFN) responsive genes (IRGs) is elevated in patients with SLE. ${ }^{2}$ We developed a robust quantitative assay to measure changes in the IFN signature, and examined the relationship between the IFN signature and measures of clinical outcome.

Methods 314 patients were randomized 1:1:1 to receive placebo, baricitinib 2- or $4 \mathrm{mg}$ once daily for 24 weeks in study JAHH. Total RNA isolated from whole blood was analyzed using a multiplex assay panel of 6 IRGs at baseline, and Weeks 2, 12, and 24. The assay was developed and optimized using RNA samples from 1760 patients with SLE enrolled in phase 3 trials of tabalumab (an anti-B cell activating factor monoclonal antibody),(2) along with healthy controls. The IFN signature assay produced a bimodal distribution.

Results $70 \%$ of patients had an elevated IFN signature at baseline. Baricitinib significantly reduced the IFN signature by Week 24 compared with placebo ( $2 \mathrm{mg}:-20 \%, 4 \mathrm{mg}:-24 \%$, p0.05), with decreases observed as early as Week 2. In patients who had a high IFN signature at baseline, baricitinib $4 \mathrm{mg}$ significantly reduced the IFN signature at Weeks 12 ($24 \%)$ and $24(-23 \%)$ compared with placebo (p0.01); decreases were also observed at Weeks 12 and 24 with baricitinib $2 \mathrm{mg}$, but the difference from placebo was not statistically significant. Baricitinib $4 \mathrm{mg}$ treatment resulted in significant clinical improvement in the resolution of arthritis or rash determined by the SLEDAI-2K. ${ }^{1}$ However, the effect of baricitinib on IFN signature reduction (change from baseline and absolute baseline value) did not correlate with SLEDAI-2K-defined clinical improvement at Week 12 or 24.

Conclusions A dose-dependent decrease in the IFN signature was observed in baricitinib-treated patients with SLE. Baricitinib treatment resulted in clinical improvement across various measures of SLE disease activity. ${ }^{1}$ Response was observed with baricitinib regardless of the change in the IFN gene signature. These data suggest that the clinical improvement observed in baricitinib-treated patients with SLE may be the result of baricitinib-mediated effects on multiple cytokine pathways that may include, but are not limited to, IFN signaling. Ongoing studies using gene arrays are now surveying global immune pathways to better characterize the mechanism of action of baricitinib in SLE.

Funding Source(s): Eli Lilly and Company

\section{REFERENCES}

1. Wallace, D. et.al. DOI:10.1136/annrheumdis-2018-eular.1918

2. Hoffman,W. et.al. ArthritisRheumatol.2017;69:643-654.

\section{NRF2 REGULATION OF THE INTERFERON SIGNATURE IN LUPUS MACROPHAGES}

${ }^{1}$ Shuhong Han, ${ }^{2}$ Haoyang Zhuang, ${ }^{3}$ Pui Lee, ${ }^{4}$ Mingjia Li, ${ }^{5}$ Peter Nigrovic, ${ }^{6}$ Westley $\mathrm{H}$ Reeves*. 'Division of Rheumatology and Clinical Immunology, University of Florida; ${ }^{2}$ 1953; ${ }^{3}$ Div. of Immunology, Boston Children's Hospital; ${ }^{4}$ University of Florida; ${ }^{5}$ Div. of Immunology, Boston Children's Hospital; ${ }^{6}$ Division of Rheumatology and Clinical Immunology, University of Florida

\subsection{6/lupus-2019-Ism.186}

Background Peripheral blood cells from two-thirds of adult lupus patients exhibit a gene expression program (interferon signature) attributed to the over-production of interferon (IFN) and other type I IFNs (IFN-I). IFN-I may be involved in the pathogenesis of lupus. Although plasmacytoid dendritic cells produce large amounts of IFN-I, our studies in an experimental lupus model suggest that macrophages and/or monocytes may play an important role in generating the interferon signature. This study sought to define how macrophages influence the interferon signature.

Methods Proinflammatory and anti-inflammatory (pro-resolving) macrophages were isolated from mice with pristaneinduced lupus and were analyzed by RNA-sequencing (RNASeq) and quantitative PCR (qPCR). Protein expression in macrophage subsets was evaluated by flow cytometry. The role of nuclear factor erythroid 2 like 2 (Nrf2) activators was examined in vivo

Results Transcriptional profiling (RNA-Seq) of CD11b+Ly6Gperitoneal macrophages from mice with experimental lupus unexpectedly indicated a strong interferon signature in proinflammatory (Ly6ChiCD138-), but not anti-inflammatory (Ly6CCD138+), macrophages exposed to the same IFN-I concentrations. Along with higher IFN-I regulated gene expression, proinflammatory macrophages expressed lower levels of genes regulated by nuclear factor erythroid 2 like 2 (Nrf2) than antiinflammatory macrophages. Transcript levels of IFN receptor 1 chain (Ifnar1), IFNAR surface staining, and mitochondrial superoxide all were higher in proinflammatory macrophages. Administration of the Nrf2 activator CDDO-Im to lupus mice decreased IFNAR expression, blocked IFN-driven Stat1 phosphorylation, and reduced IFN-regulated gene expression. Thus, the interferon signature in murine lupus critically depends on Nrf2-regulated changes in IFNAR expression in macrophages. Human peripheral blood mononuclear cells exhibited a similar pattern: high IFNAR expression in classical monocytes and lower levels in non-classical monocytes. The data suggest that anti-inflammatory macrophages/monocytes are insensitive to IFN signaling, potentially serving a role in the resolution of inflammation.

Conclusions These studies reveal that the relative abundance of different monocyte/macrophage subsets (proinflammatory 
vs. anti-inflammatory/pro-resolving) helps determine the magnitude of the interferon signature. In addition to reflecting IFNI production, the interferon signature critically depends on the Nrf2-regulated responsiveness of macrophages to signaling through the IFNAR. These studies provide a new perspective on the interferon signature that may have implications for understanding the pathogenesis of lupus. In addition, these studies suggest that $\mathrm{Nrf} 2$ activators could have a role in the therapy of lupus.

Funding Source(s): NIH

\section{APPLICATION OF TEXT MINING METHODS TO IDENTIFY LUPUS NEPHRITIS FROM ELECTRONIC HEALTH RECORDS}

${ }^{1}$ Milena A Gianfrancesco*, ${ }^{2}$ Suzanne Tamang, ${ }^{3}$ Gabriela Schmajuk, ${ }^{4}$ Jinoos Yazdany. ${ }^{1}$ Division of Rheumatology, Department of Medicine, University of California, San Francisco; ${ }^{2}$ Center for Pppulation Health Sciences, Stanford University; ${ }^{3}$ University of California, San Francisco; San Francisco VA Medical Center; ${ }^{4}$ UC San Francisco

\subsection{6/lupus-2019-|sm.187}

Background Lupus nephritis (LN), or chronic inflammation of the kidneys, is a frequent complication of SLE and associated with higher overall morbidity and mortality. Previous studies have found that LN affects individuals of Hispanic, African American and Asian descent more than those of European ancestry. Accurate estimates of $\mathrm{LN}$ in the population remain limited due to the inability to capture this information through structured data fields in electronic health records (EHRs). We aimed to develop a text mining pipeline to extract information on LN diagnosis within clinical notes in the EHR of a large, diverse university health system.

Methods Individuals with a single diagnosis code for SLE (710.0) during June 1, 2012 November 5, 2016 from the EHR of a university health system were included $(n=2,509)$. All clinical notes for patients were extracted and annotated using a clinical text-mining tool, the Clinical Event Recognizer (CLEVER), and a custom-built dictionary that included lupus nephritis and associated terms. Positive and negative mentions of $\mathrm{LN}$ were tagged and evaluated for performance.

Results Over 1300 positive and 400 negative mentions of LN were detected from clinical notes. Manual review of 50 note mentions (25 positive and 25 negative) determined that our text-mining tool detected LN with $79 \%$ sensitivity and $86 \%$ specificity (table).

Conclusions We conducted the first text-mining strategy to extract LN status from clinical notes. Additional evaluation of the LN text-mining pipeline on a gold-standard chart-reviewed cohort of SLE patients $(n=332)$ is ongoing. Further refinement of the pipeline will allow us to classify stage of LN (Class I-V) to better phenotype SLE severity, and conduct

Abstract 187 Table 1 Number of positive and negative mentions of lupus nephritis identified through the text-mining tool and manual review.

\begin{tabular}{lcc}
\hline Text-Mining Tool & \multicolumn{3}{c}{ Manual Review } \\
\hline & Positive & Negative \\
\hline Positive & 22 & 3 \\
Negative & 6 & 19 \\
Total: & 28 & 22 \\
\hline
\end{tabular}

follow-up studies to determine factors associated with this important disease outcome.

Funding Source(s): NIH-NIAMS F32 AR070585 (Gianfrancesco)

\section{VALIDATION OF AN ADMINISTRATIVE ALGORITHM TO ASSESS SLE DISEASE SEVERITY}

${ }^{1}$ Cameron B Speyer*, 'Daniel Li, ${ }^{1}$ Hongshu Guan, ${ }^{1}$ Kazuki Yoshida, ${ }^{2}$ April Jorge, ${ }^{1}$ Candace $\mathrm{H}$ Feldman, ${ }^{1}$ Karen $\mathrm{H}$ Costenbader. 'Division of Rheumatology, Immunology and Allergy, Brigham and Women's Hospital; '2Division of Rheumatology, Allergy, and Immunology, Massachusetts General Hospital

\subsection{6/lupus-2019-Ism.188}

Background SLE severity is heterogeneous: some patients have mild disease with rashes and arthritis, while others have severe multi-organ system involvement. It is challenging to study SLE in administrative databases given this heterogeneity. Garris et al developed an administrative claims-based SLE severity algorithm derived from elements of the SLEDAI, SLAM and BILAG instruments (Garris, J Med Econ 2013). It employs ICD-9, CPT and NDC claims over a 1 year period and classifies patients as having mild, moderate or severe disease. We sought to validate this administrative algorithm in comparison to SLEDAI scores at clinical visits.

Methods We identified 100 SLE patients followed at the Brigham and Womens Hospital (BWH) Lupus Center (2008-2010) with SLEDAI-2K (Gladman, J Rheumatol 2002) data at each visit over a 1 year period per person. We also obtained ICD9, CPT and NDC codes for the Garris algorithm items (e.g. codes for glucocorticoids, ICD-9 codes for pericarditis) for the same year per subject. We compared Garris SLE severity to the highest SLEDAI-2K in that year. We defined the SLEDAI$2 \mathrm{~K}$ categories of mild $<3$, moderate $3-6$, and severe $>6$ as in the literature (Polachek, Arthritis Care Res 2017). We compared classification in binary categories of mild vs. moderate/ severe and mild/moderate vs. severe. For each, we calculated sensitivity, specificity, and C-statistics.

Results We analyzed 377 SLEDAI-2K assessments on 100 subjects (mean 3.77 [SD 2.63]) in the BWH Lupus Cohort. For the Garris vs. highest SLEDAI-2K model, 56 of 100 subjects were classified similarly by Garris and highest SLEDAI-2K (23/ 36 mild, 22/34 moderate, and 11/36 severe by SLEDAI-2K). The performance characteristics compared to the highest SLEDAI-2K of the year were: C-statistics were 0.755 for mild/ moderate vs. severe SLE severity and 0.740 for mild vs. moderate/severe (table). Sensitivity of the Garris algorithm compared to the highest SLEDAI-2K were $63.9 \%$ for mild vs. moderate/severe and $94.3 \%$ for mild/moderate vs. severe.

Abstract 188 Table 1 Garris administrative algorithm for SLE severity vs. highest SLEDAI-2K

\begin{tabular}{lcc}
\hline \multicolumn{2}{c}{ Highest SLEDAI-2K } \\
\hline & $\begin{array}{c}\text { Mild vs. Moderate/ } \\
\text { Severe }\end{array}$ & $\begin{array}{c}\text { Mild/Moderate vs. } \\
\text { Severe }\end{array}$ \\
\hline Sensitivity & $63.9 \%$ & $94.3 \%$ \\
Specificity & $82.8 \%$ & $36.7 \%$ \\
C-statistic & 0.740 & 0.755 \\
\hline
\end{tabular}

